\title{
Replik zum vorangegangenen Beitrag «Swiss Medical Board - cui bono?»
}

\author{
Das Fachgremium des Swiss Medical Board hat bereits in verschiedenen Publikationen \\ und Veranstaltungen zu Kommentaren zum Bericht «Systematisches Mammogra- \\ phie-Screening» vom Februar 2014 Stellung genommen. Eine Antwort zum voran- \\ gehenden Beitrag wäre daher weitgehend eine Wiederholung früherer Aussagen. \\ In der folgenden Replik beleuchten daher Spezialisten des Instituts für Sozial- und \\ Präventivmedizin (ISPM) Bern die Thematik aus einer etwas anderen Perspektive.
}

Peter Jüni,

Tobias Erlanger,

Marcel Zwahlen

Institut für Sozial- und Präventivmedizin (ISPM), Universität Bern

\section{Korrespondenz:}

Susanna Marti Calmell Sekretariat Trägerschaft Swiss Medical Board

Stampfenbachstrasse 30

CH-8090 Zürich

Tel. 0432592479

info[at]medical-board.ch
Das Swiss Medical Board (SMB) sieht sich seit der Publikation des Berichtes «Systematisches Mammographie-Screening» mit harscher Kritik und Emotionalität konfrontiert [1]. Der vorangegangene Beitrag von Cerny, Passweg und Noseda auf S. 1726 dieser Ausgabe ist exemplarisch dafür. Wir gehen hier auf einige grundsätzliche Punkte ein, jedoch nicht auf das grundsätzliche Absprechen von fachlicher Expertise des am Bericht mitwirkenden Fachgremiums. In unserer Erfahrung folgt das SMB den Grundsätzen der evidenzbasierten Medizin und nicht denjenigen der eminenzbasierten Medizin.

Eine evidenzbasierte Debatte ist erwünscht und Kritik wird gerne als essentieller Beitrag zur Weiterentwicklung aufgenommen. Die Methoden zur Berichterstellung werden laufend verbessert und weiterentwickelt. Deshalb wurde 2014 ein Konsortium von universitären Instituten für die Berichterstellung des SMB beigezogen, dem wir zusammen mit Kolleginnen und Kollegen des Basel Institute for Clinical Epidemiology and Biostatistics und dem Institut für Pharmazeutische Medizin der Universität Basel, dem Insitut Ethique Histoire Humanités der Universität Genf und dem Institut für Epidemiologie, Biostatistik und Prävention der Universität Zürich angehören.

Die Stellungnahme des SMB wurde oft fehlinterpretiert. Die Empfehlungen des Boards basieren nicht auf der durchgeführten Kosten-Nutzen-Analyse, die unserer Ansicht nach tatsächlich mit Schwächen behaftet ist. Die Stellungnahme stützt sich in erster Linie auf die Resultate der vorhandenen randomisierten Studien, wie viele andere auch [2]. Deshalb enthält der SMB-Bericht, wie von Cerny und Kollegen richtig erwähnt, selbstverständlich keine neuen Daten. Die eigentliche Frage des SMB war, wie solide die Evidenz zugunsten des systematischen Mammographie-Screenings aufgrund der vorhandenen Studien einzustufen ist.

Ein wichtiger Faktor für die SMB-Empfehlungen war die Schwierigkeit, wie die in den randomisierten

\section{Résumé}

Dans diverses publications et manifestations, le Conseil d'experts du Swiss Medical Board s'est déjà exprimé sur un certain nombre de commentaires concernant le rapport «Dépistage systématique par mammographie» de février 2014. Une réponse à l'article ci-dessus ne serait donc qu'une répétition de déclarations antérieures. C'est pourquoi, dans cette réplique, des spécialistes de I'IMSP de Berne présentent la thématique sous un autre angle.

Les recommandations du SMB reposent principalement sur la réflexion suivante: la diminution de la mortalité par cancer du sein constatée dans les études randomisées va de pair avec un risque accru de sur-diagnostics, qui est difficile à interpréter en l'absence d'indications concernant la réduction de la mortalité globale. Les études randomisées existantes permettent de supposer que la légère diminution de la mortalité par cancer du sein est compensée par les suites des sur-traitements et d'autres causes de décès. Ainsi, même sur une période prolongée de 20 ans, la mortalité globale ne pourra probablement pas être diminuée.

Les doutes du SMB quant au bénéfice du dépistage systématique par mammographie ne sont pas isolés. Aux Etats-Unis, précurseurs du dépistage par mammographie, la coalition nationale contre le cancer du sein (NBCC) considère le rapport coûts-bénéfice d'un œil critique. Otis Brawley, directeur médical de la 
Studien beobachtete Senkung der Brustkrebsmortalität bei gleichzeitig massgeblich erhöhtem Risiko für Überdiagnosen zu interpretieren ist, wenn kein Hinweis auf eine Reduktion der Gesamtsterblichkeit vorlag. Die vorliegenden randomisierten Studien [2] lassen vermuten, dass die grundsätzlich unbestrittene leichte Senkung der Brustkrebsmortalität durch die Folgen von Überbehandlungen und kompensatorische Todesursachen wieder nivelliert wird [3-5]. Einen Hinweis darauf gibt die vor kurzem publizierte Meta-Analyse der sechs als verlässlich eingestuften randomisierten kontrollierten Studien (s. Abbildung). In diesen sechs Studien zeigt sich keine Reduktion der Gesamtsterblichkeit, obwohl die beobachtete Senkung der Brustkrebsmortalität die Gesamtsterblichkeit um rund 2\% senken müsste. Das kombinierte relative Risiko betrug 1,00, mit einem engen Vertrauensintervall von 0,97 bis 1,02 [6]. Anders ausgedrückt: Die diskrete Reduktion der Brustkrebsmortalität (Abb. oberer Teil) bedeutet nicht, dass durch Mammographie auch nur ein Leben gerettet wird, wenn sämtliche Todesursachen berücksichtigt werden (Abb. unterer Teil). Die für die Meta-Analyse verwendeten Resultate waren für einen Zeitraum von 13 Jahren nach Randomisierung. In den schwedischen randomisierten MammographieStudien machte sich eine Reduktion der Brustkrebssterblichkeit üblicherweise nach 4-6 Jahren bemerkbar und stieg bis 10-12 Jahren an, wonach sie in etwa konstant blieb [7]. Die Gesamtmortalität dürfte damit auch über einen längeren Zeitraum von $20 \mathrm{Jah}$ ren nicht gesenkt werden. Die in der Kosten-NutzenAnalyse des SMB verwendete Annahme von 13 Jahren, während denen durch die leichte Reduktion der Brustkrebsmortalität tatsächlich Leben gerettet werden, war entgegen der Argumente von Cerny et al.

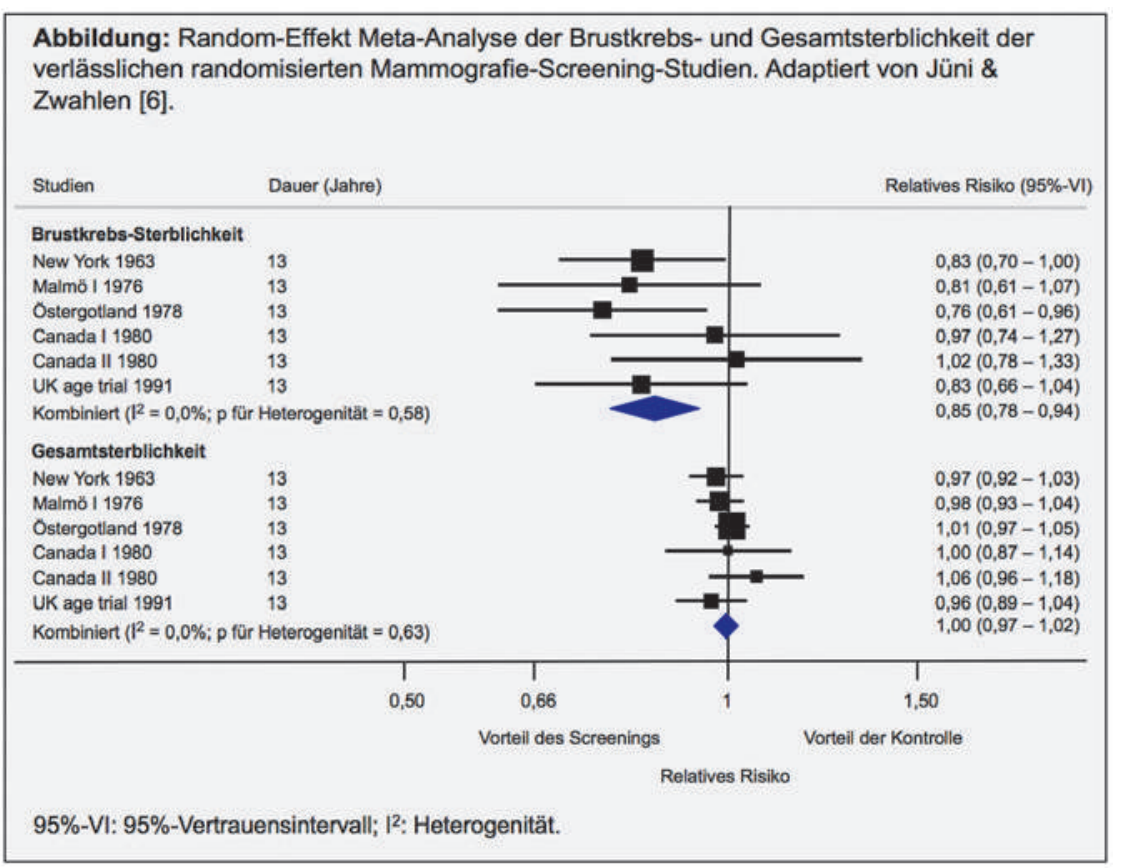

société américaine du cancer, estime que le bénéfice du dépistage par mammographie a été largement surestimé.

Malheureusement, les données à disposition ne sont pas assez convaincantes pour que les sceptiques ou les partisans du dépistage par mammographie changent d'avis. Toutefois, il y a consensus sur un point: les opinions divergent quant aux bénéfices et aux dommages de la mammographie. Dès lors, il serait important que les femmes invitées à passer une mammographie bénéficient d'une information pondérée, transparente et compréhensible sur les bénéfices (légère réduction de la mortalité par cancer du sein avec des doutes quant à la diminution de la mortalité globale) et les risques (sur-diagnostics et clarifications de résultats faux positifs).

grundsätzlich zugunsten der Mammographie. Die Meta-Analyse hätte gute Argumente für die Annahme geliefert, dass durch Mammographie gar kein Leben gerettet wird [6].

Aktuelle randomisierte Studien sind inexistent, womit sich die Frage der Übertragbarkeit der Resultate der vorliegenden randomisierten Studien auf die heutige Situation stellt. Die vorhandenen Studien rekrutierten Frauen vor 25 bis 50 Jahren - damals verwendete Diagnosetechniken und Therapien des Brustkrebses sind mit den heute verwendeten nicht mehr vergleichbar. Es scheint plausibel, dass die heute vorliegende höhere Sensitivität der Mammographien das Problem der Überdiagnose verstärkt und die verbesserte Behandlung auch von fortgeschrittenen Tumoren den Vorteil schmälert, der durch die Früherkennung zu erreichen ist $[8,9]$.

Cerny et al. bemängeln, dass keine aktuellen Erkenntnisse aus den Mammographie-Screening-Programmen berücksichtigt wurden. Beobachtungsstudien mit Daten aus laufenden Programmen erlauben es, gewisse Performance-Indikatoren zu berechnen, sind aber äusserst heikel, wenn mit ihnen der reale Effekt einer Screening-Massnahme eruiert werden soll [8]. Implizit werden in diesen Studien zumeist Screening-Teilnehmerinnen mit Nicht-Teilnehmerinnen verglichen, was zu Problemen führt (s. Kasten «Healthy Screnee»-Effekt nächste Seite) [12, 13].

Das SMB ist mit seinen Zweifeln am Nutzen der systematischen Mammographie-Screening-Programme nicht alleine. In den USA, eine der Vorreiternationen des Mammographie-Screenings, äussert sich die landesweite Koalition gegen Brustkrebs (NBCC) bezüglich des Kosten-Nutzen-Verhältnisses kritisch. Otis Brawley, ärztlicher Leiter der US-Krebsgesellschaft, meint, dass der Nutzen von Mammo- 


\section{Der «Healthy Screenee»-Effekt}

In der in den 1960er Jahren in New York durchgeführten randomisierten Studie zur Wirksamkeit des Mammographie-Screenings war das Risiko, an Brustkrebs zu sterben, bei den zur Mammographie eingeladenen Frauen um etwa 20\% geringer als in der Gruppe, die nicht eingeladen worden war. Die Sterblichkeit aufgrund anderer Ursachen war in beiden Gruppen praktisch gleich (siehe Tabelle unten). Innerhalb der zum Screening eingeladenen Gruppe gab es jedoch einen ausserordentlichen Unterschied in der Nicht-Brustkrebsmortalität zwischen Frauen, die tatsächlich an der Mammographie teilnahmen und solchen, die der Einladung zum Screening nicht gefolgt waren. Dies ist auf einen Selektionseffekt («Healthy Screenee-Effekt») zurückzuführen. Offenbar nahmen eher «gesündere» Frauen am Screening-Programm teil. Solche Selektionseffekte sind ubiquitär. Das Ausmass und die Richtung des Effekts können aber von Studie zu Studie und Land zu Land anders sein.

Nicht-Brustkrebssterblichkeit pro 10000 Lebensjahre

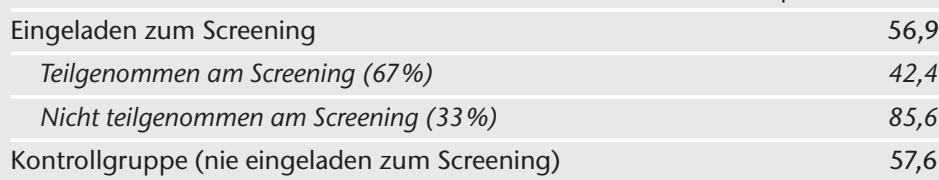

Übernommen und adaptiert von Fink 1968 [10], Shapiro 1977 [11] ständliche Information über den Nutzen (bescheidene Reduktion der Brustkrebssterblichkeit bei Unklarheit über eine Reduktion der Gesamtsterblichkeit) und die schädlichen Auswirkungen der Mammographie (Überdiagnosen und Abklärungen von falsch positiven Befunden). Daran sollten wir nun gemeinsam arbeiten.

\section{Literatur}

1 Swiss Medical Board. Systematisches MammographieScreening. Bericht vom 15. Dezember 2013.

2 Marmot MG, Altman DG, Cameron DA, et al. The benefits and harms of breast cancer screening: an independent review. Br J Cancer. 2013;108:2205-40.

3 Bleyer A, Thomas CR Jr, Baines C, Miller AB. Flawed assumptions used to defend screening mammography. Cancer. 2014 (Epub: PMCID: 25272974).

4 Kalager M, Zelen M, Langmark F, Adami HO. Effect of screening mammography on breast-cancer mortality in Norway. N Engl J Med. 2010;363:1203-10.

5 Miller AB, Wall C, Baines CJ, Sun P, To T, Narod SA. Twenty five year follow-up for breast cancer incidence and mortality of the Canadian National Breast Screening Study: randomised screening trial. BMJ. 2014;348:g366.

6 Jüni P, Zwahlen M. It is time to initiate another breast cancer screening trial. Ann Intern Med. 2014;160:864-6.

7 Nyström L, Andersson I, Bjurstam N, Frisell J, Nordenskjöld B, Rutqvist LE. Long-term effects of mammography screening: updated overview of the Swedish randomised trials. Lancet. 2002;359:909-19.

8 Berry DA, Cronin KA, Plevritis SK, Fryback DG, Clarke $\mathrm{L}$, Zelen $\mathrm{M}$ et al. Cancer Intervention and Surveillance Modeling Network (CISNET) Collaborators. Effect of screening and adjuvant therapy on mortality from breast cancer. N Engl J Med. 2005;353:1784-92.

9 Hakama M, Pokhrel A, Malila N, Hakulinen T. Sensitivity, effect and overdiagnosis in screening for cancers with detectable pre-invasive phase. Int J Cancer. 2014; [Epub: PMID: 24975995]

10 Fink R, Shapiro S, Lewison J. The reluctant participant in a breast cancer screening program. Public Health Reports. 1968;83:479-90.

11 Shapiro S. Evidence on screening for breast cancer from a randomized trial. Cancer. 1977;39:2772-82.

12 Knox G. Case-control studies of screening procedures. Public Health. 1991;105:55-61.

13 Raffle AE. Commentary: Case-control studies of screening should carry a health warning. Int J Epidemiol. 2003;32:577-8.

14 Brawley OW, Goldberg P. How we do harm. A doctor breaks ranks about being sick in America. Griffin Verlag; 2012.

15 NBCC (National Breast Cancer Coalition) Webseite besucht 21. 10. 2014: www.breastcancerdeadline2020. org/breast-cancer-information/

16 Grill M, Hackenbroch V. Unsinn in bester Qualität. Der Spiegel. 2014;30:100-4.

17 EUREF (European Reference Organisation for Quality Assured Breast Screening and Diagnostic Services). European guidelines for quality assurance in breast cancer screening and diagnosis. Fourth Edition; 2006. 